\title{
LES FERRITES POUR HYPERFRÉQUENCES
}

\author{
J. NICOLAS \\ THOMSON-CSF, Laboratoire Central de Recherches, \\ Domaine de Corbeville, 91401 Orsay, France
}

\begin{abstract}
Résumé. - Le principe général de fonctionnement des ferrites pour hyperfréquences est donné après un bref rappel sur la résonance magnétique. Les pertes de différentes origines pouvant apparaître dans ces matériaux sont considérées. On montre à quels problèmes physicochimiques est liée l'élimination des pertes diélectriques. Les pertes magnétiques sont reliées aux trois largeurs de raie expérimentalement mesurables : la largeur de raie du mode principal $\Delta H$, la largeur de raie effective $\Delta H_{\text {eff }}$ mesurée loin de la résonance, et la largeur de raie d'ondes de spins liée au seuil d'apparition des effets non linéaires de puissance. Ces différentes largeurs de raie doivent dépendre, d'une manière différente, du phénomène d'amortissement des mouvements de spins. Les matériaux utilisés actuellement aux hyperfréquences de structures grenat et spinelle sont passés en revue. Les voies de recherche paraissant actuellement les plus intéressantes sont également décrites et spécialement celles qui concernent les grenats polycristallins à très faibles largeurs de raies, des grenats très stables en température et les nouveaux spinelles à base de lithium.
\end{abstract}

Abstract. - The general mode of working of microwave ferrites is given after a short recall about the magnetic resonance. The different kinds of losses, which exist in these materials, are considered. The physico-chemical problems related to the dielectric losses are pointed out. In connection with the magnetic losses three linewidths are introduced : the resonance linewidth $\Delta H$, the effective linewidth $\Delta H_{\text {eff }}$ measured far from resonance and the spin wave linewidth related to the critical threshold field for the not linear power effects. These linewidths depend, by different ways, on the dumping of the spin motions. The materials actually used at microwave frequencies are surveyed. The objects of research, which seems now the most interesting are pointed out : polycrystalline garnets with very small resonance linewidths, garnets very stable with temperature, new lithium spinels.

1. Introduction. - Aux hyperfréquences, c'est-àdire à des fréquences comprises approximativement entre $100 \mathrm{MHz}$ et $100000 \mathrm{MHz}$, deux classes d'appareils sont réalisées avec des ferrites.

La première classe est celle des dispositifs non réciproques pour lesquels les ferrites sont pratiquement irremplaçables et qui correspond à la plus grande part des applications. Ces dispositifs sont le plus souvent des isolateurs ou des circulateurs dont les fonctions sont les suivantes. Un isolateur est simplement une section de guide d'onde dans laquelle les ondes électromagnétiques peuvent se propager dans un sens (de l'entrée vers la sortie) sans atténuation notable tandis qu'elles sont très atténuées si elles se propagent dans le sens inverse (sortie vers l'entrée). Un circulateur à $n$ voies est un appareil possédant $n$ entrées ou sorties et fonctionnant de la manière suivante (voir Fig. 1). Une onde électromagnétique entrant par la voie 1 sort par la voie 2 . Une onde entrant par la voie 2 sort par la voie 3, etc... Une onde entrant par la voie n sort par la voie 1 . Les autres possibilités sont interdites. Ces types d'appareils permettent ainsi des séparations très faciles entre différentes voies d'un circuit hyperfréquence ce qui s'avère indispensable spécialement dans la technique actuelle des radars et des faisceaux hertziens.
La deuxième classe est celle des dispositifs réci. proques ; il s'agit par exemple de déphaseurs commandables électriquement. D'autres solutions que les

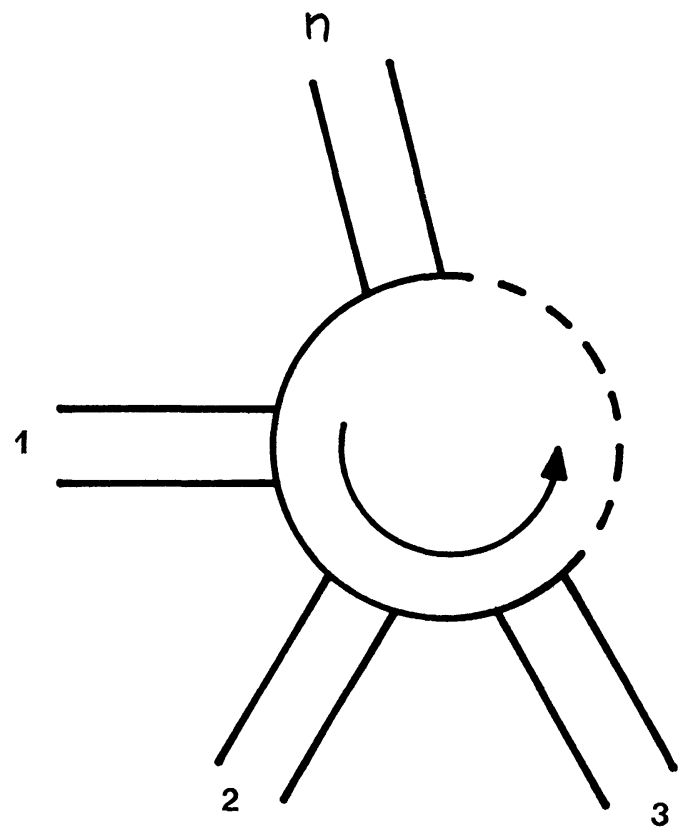

Fig. 1. - Schéma d'un circulateur à $n$ voies. 
ferrites peuvent alors exister par exemple celles utilisant des diodes à semi-conducteurs.

De toute manière on exploite aux hyperfréquences une perméabilité du matériau ferrite qui est sous la dépendance du phénomène gyromagnétique. Il en résulte que cette perméabilité dépend comme nous le verrons du champ magnétique statique appliqué (base des effets de déphasage à commande électrique) et de la polarisation de l'onde électromagnétique par rapport à ce champ statique (base des effets de nonréciprocité). Les caractéristiques à considérer sont les caractéristiques diélectriques et magnétiques. Ces dernières sont très liées au phénomène de résonance gyromagnétique par rapport auquel on peut définir un champ magnétique statique de résonance et une largeur de raie. On introduit également une largeur de raie effective caractérisant le matériau loin de la résonance et une largeur de raie d'onde de spins liée à l'apparition d'effets non linéaires au-delà d'un champ hyperfréquence seuil. Ces deux grandeurs traduisent l'amortissement du mouvement de l'aimantation.

Nous montrerons que les ferrites pour hyperfréquences doivent présenter une aimantation adaptée à la fréquence d'utilisation. Or l'aimantation peut être ajustée d'une manière très précise sur la base de la théorie du ferrimagnétisme de L. Néel. Aux possibilités offertes par les ferrites de structure spinelle sont venues s'ajouter celles des ferrites de structure grenat découverts par Bertaut et Forrat en 1956. Ces corps sont devenus irremplaçables pour la plupart des applications au-dessous de $5000 \mathrm{MHz}$. Nous essaierons de montrer comment, à partir de diagrammes des ferrites de structures spinelle ou grenat, sont obtenues les caractéristiques nécessaires pour les applications. Nous essaierons également de dégager les problèmes actuels et l'évolution de la recherche dans ce domaine.

2. Principe général de fonctionnement des ferrites pour hyperfréquences. - Nous décrirons d'abord brièvement le phénomène de résonance magnétique. Considérons un ellipsoïde d'axes a, b, c (suivant $o x, o y, o z$ ) constitué par un matériau magnétique. Cet ellipsoïde est plongé dans un champ magnétique statique $\mathbf{H}_{\mathbf{a}}$ uniforme avant l'introduction du matériau, ayant la direction $o z$. On sait qu'il en résulte un champ à l'intérieur du matériau $\mathbf{H}_{\mathrm{int}}$ uniforme dirigé suivant oz. $\mathbf{H}_{\mathrm{int}}$ est la somme de $\mathbf{H}_{\mathrm{a}}$ et du champ démagnétisant, uniforme à l'intérieur de l'échantillon :

$$
\mathbf{H}_{\text {int }}=\mathbf{H}_{\mathrm{a}}-N_{z} \frac{\mathbf{I}_{\mathrm{s}}}{\mu_{0}}
$$

$N_{z}$ étant le facteur démagnétisant dans la direction $o z$.

On suppose que ce champ statique est suffisant pour saturer le matériau dont l'aimantation $\mathbf{I}_{\mathbf{s}}$ est dirigée suivant $o z$. De plus, on applique au matériau un champ hyperfréquence h perpendiculaire à $o z$ donc situé dans le plan $o x$, oy et tournant dans ce plan avec la pulsation $\omega=2 \pi f$. Le champ correspondant à l'intérieur $\mathrm{du}$

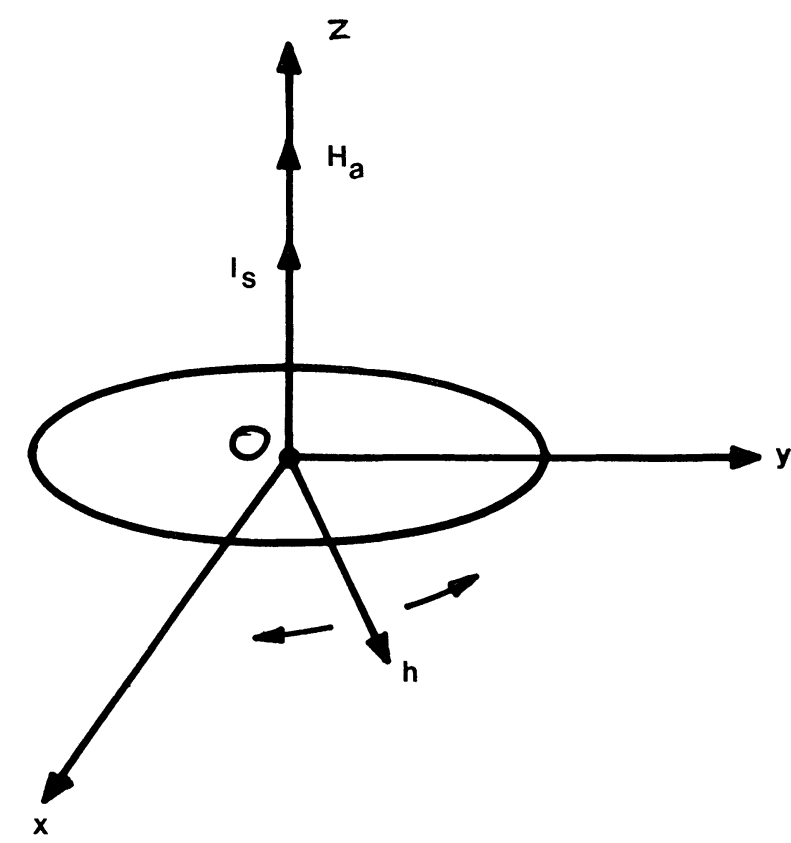

FIG. 2. - Ellipsoïde de matériau magnétique soumis à un champ statique $\mathbf{H}$ et un champ hyperfréquence $\mathbf{h}$, tournant dans le plan $x o y$.

matériau est noté $\mathbf{h}_{\text {int }}$ et il est supposé d'intensité suffisamment petite devant $H_{\text {int }}$. Le vecteur d'aimantation devient I tel que :

$$
\mathbf{I}=\mu_{0} \chi_{\text {int }}\left(\mathbf{H}_{\text {int }}+\mathbf{h}_{\text {int }}\right)
$$

$\mu_{0}$ étant la perméabilité du vide et $\chi_{\text {int }}$ la susceptibilité magnétique du matériau. Cette susceptibilité peut être explicitée en analysant le mouvement de l'extrémité du vecteur I. Ceci peut se faire en appliquant aux porteurs de moment magnétique, par exemple les électrons, le théorème du moment cinétique :

$\frac{1}{\gamma} \frac{\mathrm{d} \mathbf{I}}{\mathrm{d} t}=\mathbf{I} \wedge\left(\mathbf{H}_{\mathrm{int}}+\mathbf{h}_{\mathrm{int}}\right)+$ terme d'amortissement

$\gamma$ est le rapport gyromagnétique, c'est-à-dire le rapport entre le moment cinétique et le moment magnétique. On a pratiquement $\gamma=1,105 \times 10^{5} g_{\text {eff }}(\mathrm{m} / \mathrm{A}-\mathrm{s}) \cdot g_{\text {eff }}$ est le facteur gyromagnétique égal à 2 pour l'électron libre.

La résolution des équations (2) et (3) dans laquelle on choisit un terme d'amortissement convenable s'effectue en ne s'intéressant qu'aux composantes dépendant du temps. En fait, on considère, parce qu'intervenant le plus directement dans les problèmes réels, une susceptibilité $\chi$ définie par rapport au champ appliqué $\mathbf{h}$ (et reliée à $\chi_{\text {int }}$ en tenant compte de l'effet du champ démagnétisant). On trouve que la valeur de $\chi$ dépend de la polarisation de $\mathbf{h}$. On notera les suscepti-

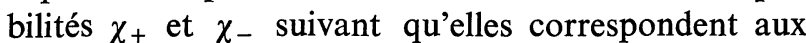
sens de rotation positif ou négatif dans le plan $o x$, oy. 
Pour tenir compte du déphasage entre l'induction et le champ, $\chi_{+}$et $\chi$ - sont des quantités complexes :

$$
\begin{aligned}
& \chi_{+}=\chi_{+}^{\prime}-j \chi_{+}^{\prime \prime} \\
& \chi_{-}=\chi_{-}^{\prime}-j \chi_{-}^{\prime \prime} .
\end{aligned}
$$

On définit aussi les perméabilités correspondantes :

$$
\mu_{+}=1+\chi_{ \pm} \text {. }
$$

La figure 3 donne l'allure des variations observées de $\mu_{+}^{\prime}, \mu_{-}^{\prime}, \mu_{+}^{\prime \prime}, \mu_{-}^{\prime \prime}$ en fonction du champ appliqué $H_{\mathrm{a}}$ à la fréquence $f$ fixe. Dans cette figure les valeurs positives

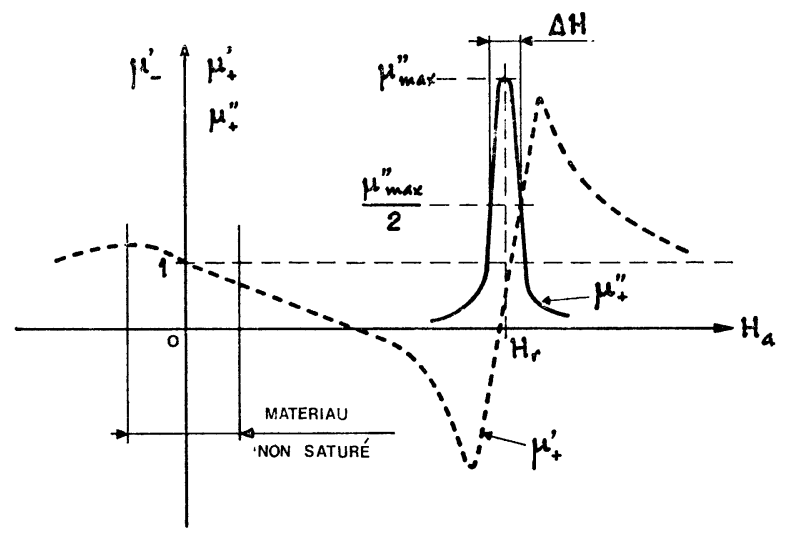

FIG. 3. - Allure des parties réelles et imaginaires des perméabilités $\mu_{+}$et $\mu_{-}$en fonction de l'intensité du champ statique $H_{\mathrm{a}}$. $\mu_{+}$est porté suivant les abscisses positives et $\mu_{-}$suivant les abscisses négatives.

de $H_{\mathrm{a}}$ correspondent à la polarisation positive pour $\mathbf{h}$ et les valeurs négatives de $H_{\mathrm{a}}$ à la polarisation négative pour $\mathbf{h}$. Les formes des courbes de $\mu_{+}^{\prime}$ et $\mu_{+}^{\prime \prime}$ sont typiques d'un phénomène de résonance. Le champ de résonance $H_{\mathrm{r}}$ est donné par la formule de Kittel :

$$
\begin{aligned}
\omega^{2}=\gamma\left[H_{\mathrm{r}}-\left(N_{z}-N_{x}\right)\right. & \left.\frac{I_{\mathrm{s}}}{\mu_{0}}\right] \times \\
\times & {\left[H_{\mathrm{r}}-\left(N_{z}-N_{y}\right) \frac{I_{\mathrm{s}}}{\mu_{0}}\right] }
\end{aligned}
$$

où $N_{x}, N_{y}$ et $N_{z}$ sont les facteurs démagnétisants de l'ellipsoïde dans les directions $o x, o y, o z$

$$
\left(N_{x}+N_{y}+N_{z}=1\right) \text {. }
$$

On définit la largeur de raie de résonance $\Delta H$ comme la largeur à mi-hauteur de la courbe de $\mu^{\prime \prime}$ en fonction de $H_{\mathrm{a}}$. On voit sur la figure 3 qu'il est possible de trouver des valeurs de $\left|H_{\mathrm{a}}\right|$ telles que les perméabilités $\mu_{+}^{\prime}$ et $\mu_{-}^{\prime}$ sont assez différentes tandis que $\mu_{+}^{\prime \prime}$ et $\mu_{-}^{\prime \prime}$ sont très faibles. C'est cette propriété qui est le plus souvent utilisée dans la construction des dispositifs non réciproques. Le champ $H_{\mathrm{a}}$ appliqué peut être soit inférieur au champ de résonance $H_{\mathrm{r}}$ soit supérieur à $H_{\mathrm{r}}$. On préfère autant que possible la première solution ne serait-ce que pour des raisons économiques, les aimants nécessaires étant moins importants. Cepen- dant, il faut tenir compte du fait que, conformément à la formule (6), le champ de résonance diminue lorsque la fréquence diminue; pratiquement au-dessous de $1000 \mathrm{MHz}$ il n'est plus possible de trouver avant la résonance des champs $H_{\mathrm{a}}$ où $\mu^{\prime \prime}$ est négligeable et l'on utilise alors des champs $H_{\mathrm{a}}$ supérieurs à $H_{\mathrm{r}}$.

Dans certains cas, par exemple celui des isolateurs à la résonance, on polarise le matériau avec un champ $H_{\mathrm{a}}$ voisin de $H_{\mathrm{r}}$ et on utilise directement la différence entre les pertes magnétiques (données par $\mu^{\prime \prime}$ ) pour les deux sens de polarisation de l'onde électromagnétique.

3. Les pertes dans les ferrites pour hyperfréquences. - Une des préoccupations majeures dans la construction des appareils à ferrite est la réduction des pertes d'insertion. Or les pertes dans les ferrites ont deux origines, diélectrique ou magnétique, que nous allons examiner successivement.

3.1 Pertes DiÉLeCtriques. - Les pertes diélectriques existent dans les ferrites pour hyperfréquences principalement à cause de l'existence de petites quantités de fer divalent à la place de fer trivalent. Ce défaut se traduit par un excès d'électrons qui, pouvant sauter d'un ion à l'autre, sont responsables d'une certaine conduction et de pertes diélectriques. Aussi, l'absence de pertes diélectriques est essentiellement une question de perfection. Cela signifie généralement un matériau pur, homogène, possédant une seule phase ayant exactement la proportion des ions de la formule chimique. La perfection absolue n'est jamais possible mais des matériaux suffisamment proches de cette perfection peuvent être réalisés en prenant des précautions suffisantes en ce qui concerne les compositions chimiques et la technologie. D'abord il est préférable de ne pas choisir des compositions nécessitant la mise en œuvre d'un élément volatil car la proportion rigoureuse des ions de la formule chimique théorique est difficile à obtenir dans ces conditions. Si ce n'est pas possible on s'efforce de réaliser la synthèse à une température suffisamment basse pour ne pas avoir de trop sérieux problèmes. Un bon exemple de cette situation sera donné à propos des ferrites de structure spinelle contenant du lithium.

La préparation des ferrites de structure grenat ayant 'de très faibles pertes diélectriques demande un ajustement particulièrement précis des proportions des différents ions métalliques. Ceci nécessite de tenir soigneusement en compte la quantité de fer introduite par les broyages. Les proportions finales des différents ions métalliques doivent être ajustées à environ $10^{-3}$. En plus, compte tenu des problèmes de reproductibilité sous-jacents à toute fabrication, le traitement thermique de synthèse doit être précis. La température maximum doit être définie et reproductible à environ $5^{\circ} \mathrm{C}$. Dans ces conditions il est possible de produire des greñats avec une tangente d'angle de perte inférieure à $10^{-4}$ à $10000 \mathrm{MHz}$. Ceci est généralement suffisamment faible pour ne donner lieu qu'à des pertes assez négligeables devant les autres causes de pertes dans les dispositifs. 
3.2 Pertes magnétiQues a bas niveau de puissance HYPERFRÉQUENCE. - Les courbes de la figure 3 sont d'allure lorentzienne et doivent donc répondre à l'expression :

$$
\mu_{ \pm}=1+\frac{I_{\mathrm{s}} / \mu_{0}}{H_{\mathrm{a}} \mp H_{\mathrm{r}}+j \frac{1}{2} \Delta H} .
$$

C'est bien à une expression de cette forme qu'aboutit la résolution des relations (2) et (3).

Expérimentalement les choses sont cependant un peu plus compliquées. Pour des champs appliqués assez éloignés de la résonance ce qui correspond à la plupart des applications, l'expression (7) est valable mais à condition de remplacer la largeur de raie $\Delta H$ par une largeur de raie plus faible dite largeur de raie effective $\Delta H_{\text {eff }}$. En pratique la valeur de $\Delta H_{\text {eff }}$ permet de calculer les pertes magnétiques. Inversement la mesure de $\mu_{ \pm}^{\prime}$ et $\mu_{ \pm}^{\prime \prime}$ à champ $H_{\mathrm{a}}$ donné, donne la valeur de $\Delta H_{\text {eff }}$ par l'expression :

$$
\Delta H_{\text {eff }}=\frac{I_{\mathrm{s}}}{\mu_{0}} \operatorname{Im}\left(\frac{1}{\chi_{ \pm}}\right)
$$

où $\operatorname{Im}\left(1 / \chi_{ \pm}\right)$signifie la partie imaginaire de $1 / \chi_{ \pm}$. On constate que $\Delta H_{\text {eff }}$ ainsi mesuré est constant loin de la résonance.

$\mathrm{Au}$ contraire, au voisinage de la résonance, il existe des causes d'élargissement de la raie qui ne sont pas à proprement parler liées à l'amortissement du mouvement des porteurs de moment magnétique. Il s'agit surtout de l'influence des champs démagnétisants locaux (dus à l'existence de pores dans le matériau) et à l'influence de l'anisotropie magnétocristalline (traduisant le fait que le champ de résonance dans un monocristal dépend de la direction du champ appliqué par rapport aux axes cristallographiques). Une expression de $\Delta H$ couramment admise met en évidence cet élargissement étudié par Schlömann, et qui correspond assez bien à la réalité :

$$
\Delta H=\Delta H_{\mathrm{int}}+1,5 p \frac{I_{\mathrm{s}}}{\mu_{0}}+\frac{K_{1}}{I_{\mathrm{s}}}
$$

où $\Delta H_{\text {int }}$ est une largeur de raie intrinsèque ; $p$ est la porosité (fraction des inclusions non magnétiques par rapport au volume total) ; $K_{1}$ est la constante d'anisotropie magnétocristalline du premier ordre.

Ainsi tandis que $\Delta H_{\text {eff }}$ détermine les pertes magnétiques, la valeur de $\Delta H$ n'intervient que dans la détermination de la "faisabilité » de l'appareil. Un faible $\Delta H$ permet de réaliser un dispositif donné plus facilement et dans une plus large bande de fréquence. Ainsi, il y a intérêt à réaliser, bien que pour des raisons différentes, des matériaux ayant un faible $\Delta H_{\text {eff }}$ et un faible $\Delta H$.

Pour obtenir un faible $\Delta H_{\text {eff }}$ il faut réduire autant que possible l'amortissement du mouvement des porteurs de moment magnétique. Il faut pour cela éliminer de la composition les ions magnétiques relaxants. Dans la pratique il suffit de n'avoir par exemple comme ions magnétiques uniquement des ions fer trivalents qui ne relaxent que très peu à cause de leur configuration électronique : couche extérieure $3 \mathrm{~d}$ à moitié pleine donc 5 spins électroniques et pas de moment orbital, donc pas de couplage spin-orbite. Effectivement les ferrites ne possédant que des ions fer trivalent en tant qu'ions magnétiques : par exemple le grenat d'yttrium $\mathrm{Y}_{3} \mathrm{Fe}_{5} \mathrm{O}_{12}$ ou le ferrite de lithium $\mathrm{Li}_{1 / 2} \mathrm{Fe}_{5 / 2} \mathrm{O}_{4}$ ont de très faibles $\Delta H_{\text {eff }}(1$ à $3 \mathrm{Oe})$. Malheureusement cette propriété n'est pas toujours compatible avec d'autres caractéristiques recherchées particulièrement une bonne stabilité en température et des compromis sont à cet égard à rechercher dans la pratique, comme nous le verrons plus loin.

Pour obtenir un faible $\Delta H$, il faut d'après l'expression (9) avoir un faible $\Delta H_{\text {int }}$ que nous verrons très relié à $\Delta H_{\text {eff }}$. Il faut aussi réduire autant que possible la porosité, ce qui est un problème technologique. Il faut enfin avoir une faible anisotropie magnétocristalline ce qui impose le choix de la structure cristallographique et de la formule chimique.

3.3 Pertes magnétiques a haut niveau de puisSANCE HYPERFRÉQUENCE. - Tout ce que nous venons de voir est valable tant que le champ magnétique hyperfréquence est suffisamment petit. Au-delà d'un certain champ critique apparaissent des phénomènes non linéaires se traduisant par des pertes magnétiques supplémentaires rapidement prohibitives dans les dispositifs. On peut distinguer parmi ces effets non linéaires ceux du premier ordre qui apparaissent généralement à des champs $H_{\mathrm{a}}$ inférieurs au champ de résonance $H_{\mathrm{r}}$ et ceux du deuxième ordre qui affectent la résonance ellemême. Les effets du premier ordre ont un seuil plus bas que ceux du deuxième ordre et ils se produisent dans une zone de champs magnétiques statiques très souvent utilisée dans les applications. Ce sont donc les plus gênants en pratique et nous allons les examiner plus spécialement ici.

Les effets non linéaires sont dus d'après Suhl au fait que les spins électroniques, porteurs de moments magnétiques, ne restent pas parallèles entre eux dans

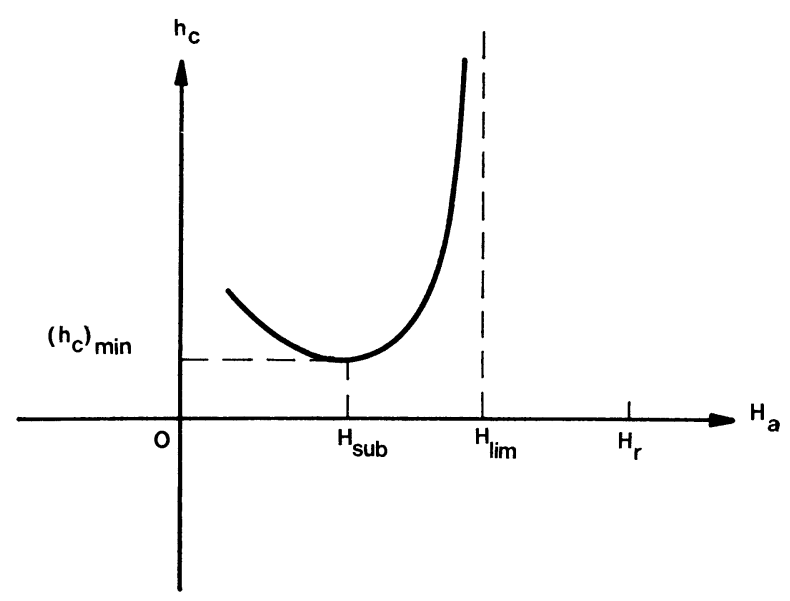

FIG. 4. - Champ critique d'effets non linéaires du $1^{\text {er }}$ ordre en fonction du champ statique $H_{\mathrm{a}}$. 
leurs mouvements et que se produisent des ondes de spins. Le champ critique hyperfréquence $h_{\mathrm{c}}$ à partir duquel de tels effets apparaissent est fonction du champ statique appliqué comme le montre la figure 4 . $h_{\mathrm{c}}$ passe par un minimum pour le champ subsidiaire $H_{\text {sub }}$ et tend vers une valeur très grande pour le champ limite $H_{1 \mathrm{im}}$. La théorie de Suhl est en bon accord avec les résultats expérimentaux. L'existence des ondes de spins est liée à l'amortissement du mouvement des spins. Ces ondes se produisent d'autant plus facilement que ce mouvement est moins amorti. Pour caractériser cet amortissement on introduit une largeur de raie d'ondes de spins $\Delta H_{\mathrm{k}}$ et l'on a, dans le cas d'un échantillon sphérique de mesure :

$$
\left(h_{\mathrm{c}}\right)_{\min }=\Delta H_{\mathrm{k}} \frac{1-\omega_{0} / \omega}{1-\frac{\omega_{M}}{2 \omega}+\sqrt{1+\frac{\omega_{M}^{2}}{4 \omega^{2}}}} \frac{2 \omega}{\omega_{M}}
$$

$\operatorname{avec} \omega_{0}=\gamma H_{\mathrm{a}}$ et $\omega_{\mathrm{M}}=\gamma \frac{I_{\mathrm{s}}}{\mu_{0}}$

$h_{\mathrm{c}}$ est dans cette formule la longueur du vecteur champ hyperfréquence tournant dans le plan xoy (polarisation circulaire).

Par contre les appareils tels que les circulateurs utilisent plutôt des disques plats de ferrite où le champ critique $\left(h_{\mathrm{c}}\right)_{\min }$ est donné approximativement, dans les cas courants, par:

$$
\left(h_{\mathrm{c}}\right)_{\min } \simeq 0,5 \Delta H_{\mathrm{k}} \frac{\omega}{\omega_{\mathrm{M}}}
$$

Le champ limite étant donné par :

$$
H_{1 \mathrm{im}}=\frac{\omega}{2 \gamma}+N_{z} \frac{I_{\mathrm{s}}}{\mu_{0}} .
$$

Ainsi dans les dispositifs où circule une certaine puissance hyperfréquence, il existe des risques d'apparition d'effets non linéaires entraînant des pertes magnétiques supplémentaires. Pour éviter cet inconvénient on peut utiliser les moyens suivants :

a) On peut accroître $h_{\mathrm{c}}$ et donc $\Delta H_{\mathrm{k}}$. Ceci peut se faire en introduisant dans le matériau des ions magnétiques relaxants, provoquant l'amortissement des mouvements de spins. Mais il en résulte, comme nous l'avons vu au chapitre précédent, un accroissement de $\Delta H_{\text {eff }}$ et donc des pertes magnétiques et des pertes d'insertion dans le dispositif. Ceci est d'autant plus gênant dans les dispositifs de puissance que ces pertes d'insertion correspondent à une énergie perdue sous forme de chaleur ce qui accroît la température du matériau et complique encore le problème.

b) Il existe une possibilité d'accroître $\Delta H_{\mathrm{k}}$ tout en ayant une augmentation de $\Delta H_{\text {eff }}$ plus modérée que dans le cas précédent en jouant sur la structure granulaire du matériau. En effet si l'on réduit suffisamment la taille des cristallites, par exemple jusque vers un micron, les conditions de propagation des ondes de spins sont perturbées et l'on constate une forte augmentation de $\Delta H_{\mathrm{k}}$. Malheureusement cette voie nécessite une technologie très particulière, celle du pressage à chaud qui est difficile et chère et on ne la réserve actuellement qu'à la résolution de problèmes particuliers, si l'on ne peut faire autrement.

c) On applique au matériau un champ statique $\boldsymbol{H}_{\mathrm{a}}$ plus grand que $\boldsymbol{H}_{\mathrm{lim}}$ pour éviter les effets non linéaires du premier ordre. Il est alors avantageux de pouvoir réaliser les dispositifs avec des champs inférieurs au champ de résonance. Des réalisations pratiques ont été faites pour la première fois à la THOMSON-CSF de circulateurs à jonction de très hautes performances basés sur ce principe à des fréquences comprises entre 5000 et $20000 \mathrm{MHz}$. Evidemment si le champ hyperfréquence est trop important des effets non linéaires du premier ordre peuvent intervenir et cette solution n'est plus valable ; les solutions $a$ ) ou $b$ ) doivent alors être mises en œuvre.

3.4 L'AMORTISSEMENT MAGNÉTIQUE. - Nous avons introduit précédemment trois largeurs de raie reliées au phénomène d'amortissement des mouvements de spins : la largeur de raie intrinsèque $\Delta H_{\text {int }}$ qui est (voir formule (9)) une des composantes de la largeur de raie du mode principal $\Delta \boldsymbol{H}$, la largeur de raie effective $\Delta H_{\text {eff }}$ définie loin de la résonance et la largeur de raie d'ondes de spins $\Delta H_{\mathrm{k}}$ décrivant l'amortissement des ondes de spins. Inglebert, dans notre laboratoire a cherché comment l'introduction d'ions à relaxation rapide $\left(\mathrm{Co}^{2+}\right.$ et terres rares magnétiques) influençait ces différentes largeurs de raie. Il a été montré qu'à la température ambiante les accroissements $\delta\left(\Delta H_{\text {int }}\right)$ et $\delta\left(\Delta H_{\text {eff }}\right)$ de $\Delta H_{\text {int }}$ et $\Delta H_{\text {eff }}$ étaient égaux et s'identifiaient également aux accroissements de $\Delta H$ observés dans les monocristaux dopés par les mêmes ions. Une relation linéaire a été expérimentalement trouvée entre $\delta\left(\Delta H_{\text {eff }}\right)$ et $\delta\left(\Delta H_{\mathrm{k}}\right)$ :

$$
\delta\left(\Delta H_{\text {eff }}\right)=A \delta\left(\Delta H_{\mathrm{k}}\right)
$$

où $A$ ne semble dépendre que de la nature de l'ion relaxant. Suivant cet ion $\boldsymbol{A}$ a une valeur comprise entre 2,5 et 3,5 alors qu'une théorie simple donne la valeur 2 . A la température ambiante les valeurs de $\delta\left(\Delta H_{\text {eff }}\right)$ sont l'objet d'une théorie de Kittel qui introduit un temps de relaxation $\tau$ pour l'ion magnétique considéré. Inglebert a pu donner dans le cadre de cette théorie des ordres de grandeur de $\tau$ pour quelques terres rares que le tableau I reproduit.

\section{TABLEAU I}

$$
\begin{array}{ccccc}
\text { Terres rares } & \text { Tb } & \text { Dy } & \text { Ho } & \frac{\mathrm{Er}}{2} \\
\tau\left(\times 10^{13} \mathrm{~s}\right) & \frac{6}{2} & \frac{-}{3} & \frac{3}{2}
\end{array}
$$

Un certain crédit peut être attribué à cette théorie qui explique très bien d'autre part les variations du champ de résonance (ou ce qui revient au même de $g_{\text {eff }}$ ) avec introduction des ions relaxants. 
L'interprétation plus approfondie des valeurs de ces temps de relaxation se heurte par contre à d'assez grosses difficultés. L'origine du phénomène d'amortissement en cause est à rechercher dans les couplages spins-orbites. La non-équivalence des sites dodécaédriques de la structure grenat peut être un obstacle à une interprétation précise.

4. Rôle de l'aimantation. - Le rôle de l'aimantation est fondamental d'abord parce qu'elle vient en facteur de tous les termes de susceptibilité magnétique comme le montre l'expression (7). L'aimantation conditionne directement l'efficacité du matériau. On serait donc tenter de penser qu'il faut toujours choisir un ferrite ayant la plus grande aimantation possible. En fait nous allons voir qu'une autre condition empêche de le faire spécialement pour les dispositifs, les plus nombreux, dans lesquels on utilise un champ statique inférieur au champ de résonance. Cela tient au fait qu'en l'absence de champ extérieur appliqué existe en chaque point du matériau un champ interne qui a principalement deux origines : d'une part l'anisotropie magnétocristalline, d'autre part les interactions dipolaires magnétiques. Ainsi un phẻnomène de résonance magnétique (dite naturelle) est possible dans ces champs statiques internes entraînant des pertes magnétiques dans le matériau. Il est important de voir à quelles fréquences ces pertes existent. En fait dans les matériaux qui nous intéressent, le champ équivalent d'anisotropie est le plus souvent faible devant les champs d'origine dipolaire. Ces derniers possèdent toute une gamme de valeurs variables d'un point à l'autre, la plus grande valeur possible étant $I_{\mathrm{s}} / \mu_{0}$. On constate qu'effectivement il existe dans tout matériau magnétique des pertes magnétiques à champ statique nul jusqu'à une fréquence limite $f_{1}$ donnée par

$$
\omega_{1}=2 \pi f_{1}=\gamma \frac{I_{\mathrm{s}}}{\mu_{0}} \text {. }
$$

Pour une fréquence $f<f_{1}$ ces pertes magnétiques existant à l'état désaimanté diminuent progressivement avec l'application d'un champ magnétique statique mais ne disparaissent pratiquement qu'avec un champ $H_{1}$, comme montré dans la figure 5 .

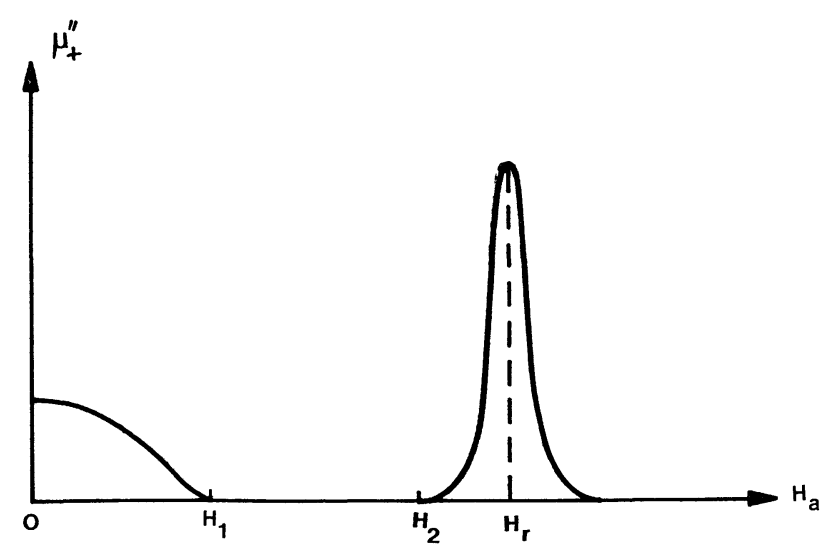

Fig. 5. - Pertes magnétiques aux champs faibles et à la résonance (pour $f$ un peu inférieur à $f_{1}=\gamma I_{\mathrm{s}} / 2 \pi$ ).
Aussi il ne sera possible de réaliser un dispositif avec un champ statique inférieur à $H_{\mathrm{r}}$ que si un intervalle suffisant de champ magnétique $\left(H_{1}, H_{2}\right)$ correspondant à des pertes magnétiques faibles, existe réellement, comme dans le cas de la figure 5. On trouve que cette condition n'est réalisée que lorsque l'aimantation n'est pas très supérieure à celle correspondant à la formule (14) où $f_{1}$ est pris comme la fréquence la plus basse à laquelle doit fonctionner le dispositif concerné. On voit aussi pourquoi les ferrites pour hyperfréquences doivent posséder une aimantation adaptée, et approximativement proportionnelle à la fréquence d'utilisation. Ceci n'est évidemment pas valable pour les matériaux utilisés dans des dispositifs utilisant un champ magnétique statique supérieur au champ de résonance, ce qui est souvent le cas à des fréquences inférieures à $1000 \mathrm{MHz}$.

5. Grenats pour hyperfréquences $\left(^{*}\right)$. -5.1 GRENATS CLASSIQUES. - Avec des moments magnétiques compris entre $200 \mathrm{G}$ et $1900 \mathrm{G}$ et de faibles largeurs de raie, ces matériaux sont bien adaptés à la réalisation des dispositifs à des fréquences comprises entre quelques centaines de $\mathrm{MHz}$ et $5000 \mathrm{MHz}$. Ils sont dérivés du grenat d'yttrium $\mathrm{Y}_{3} \mathrm{Fe}_{5} \mathrm{O}_{12}$ qui a usuellement les caractéristiques suivantes :

$$
\left.\begin{array}{l}
4 \pi M_{\mathrm{s}}=1770 \mathrm{G}\left(I_{\mathrm{s}}=0,177 \mathrm{~Wb} / \mathrm{m}^{2}\right) \\
\theta_{\mathrm{c}} \text { (point de Curie) }=280{ }^{\circ} \mathrm{C} \\
g_{\text {eff }}=2 \\
\Delta H=45 \mathrm{Oe}\left(3,6 \times 10^{3} \mathrm{~A} / \mathrm{m}\right) \\
\Delta H_{\mathrm{k}}=1,2 \text { Oe }\left(9,55 \times 10^{2} \mathrm{~A} / \mathrm{m}\right) \\
\varepsilon^{\prime}(\text { Cte diélectrique relative })=15,4 \\
\operatorname{tg} \delta_{\varepsilon}<10^{-4}
\end{array}\right\}
$$

Pour obtenir différentes valeurs d'aimantation des substitutions sont faites dans le grenat d'yttrium. Les ions fer sont placés dans deux sortes de sites cristallographiques : pour une formule chimique il y a trois ions $\mathrm{Fe}^{3+}$ dans des sites tétraédriques et deux ions $\mathrm{Fe}^{3+}$ dans des sites octaédriques. D'après la théorie du ferrimagnétisme de Néel, l'aimantation $I_{\mathrm{s}}$ est la différence entre les aimantations des sous-réseaux magnétiques. De ce fait il existe deux moyens très classiques pour diminuer $4 \pi M_{\mathrm{s}}$ :

a) Le premier moyen consiste à substituer aux ions fer, des ions aluminium non magnétiques qui vont surtout se loger dans les sites tétraédriques. Cela fait décroître le point de Curie et accroître le coefficient de température de $4 \pi M_{\mathrm{s}}$. La figure 6 donne $4 \pi M_{\mathrm{s}}$, $\Delta H$ et le coefficient de température de $4 \pi M_{\mathrm{s}}$ (entre -40 et $+85^{\circ} \mathrm{C}$ ) pour de tels matériaux de formule chimique $\mathrm{Y}_{3} \mathrm{Fe}_{5-5 y} \mathrm{Al}_{5 y} \mathrm{O}_{12}$, en fonction de la substi-

(*) Dans ce chapitre et les suivants nous utiliserons plutôt les unités uemcgs pour faciliter les comparaisons avec les données de la littérature, alors que dans les chapitres précédents les formules étaient données dans le système MKSA. 


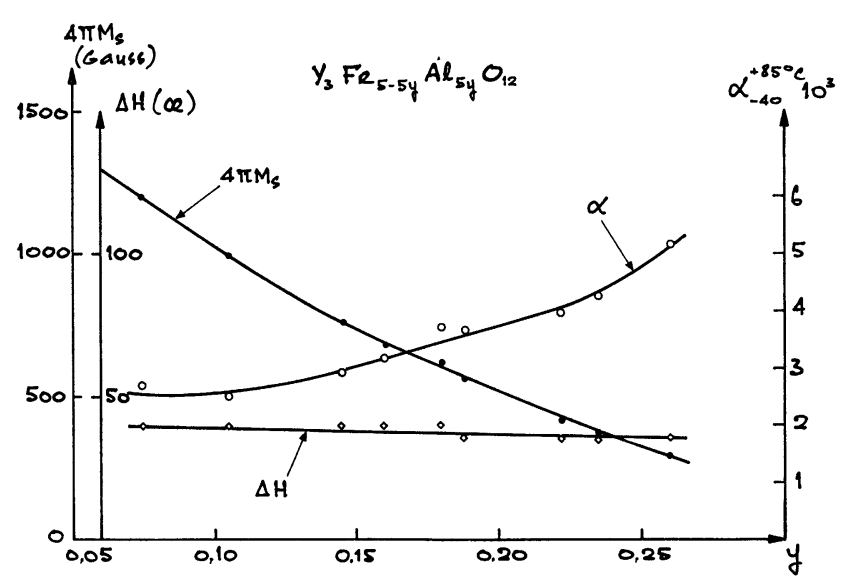

FIG. 6. - Propriétés des grenats classiques d'yttrium substitués à l'aluminium.

tution $y$ d'aluminium. Le coefficient de température $\alpha_{T_{2}}^{T_{1}}$ de $4 \pi M_{\mathrm{s}}$ est défini comme suit :

$$
\alpha_{T_{2}}^{T_{1}}=\frac{1}{T_{2}-T_{1}} \frac{\left(4 \pi M_{\mathrm{s}}\right)_{\max }-\left(4 \pi M_{\mathrm{s}}\right)_{\min }}{\left(4 \pi M_{\mathrm{s}}\right)_{20{ }^{\circ} \mathrm{C}}} .
$$

Pour tous ces matériaux sans ions magnétiques relaxants la largeur de raie d'ondes de spins est faible, comprise entre 1,5 et $2,5 \mathrm{Oe}$.

b) Le second moyen consiste à substituer dans les sites dodécaédriques à des ions yttrium, non magnétiques, des ions gadolinium dont l'aimantation vient s'apposer à la résultante de l'aimantation des ions fer. On a aussi des produits de composition

$$
\mathrm{Y}_{3-3 x} \mathrm{Gd}_{3 x} \mathrm{Fe}_{5} \mathrm{O}_{12} \text {. }
$$

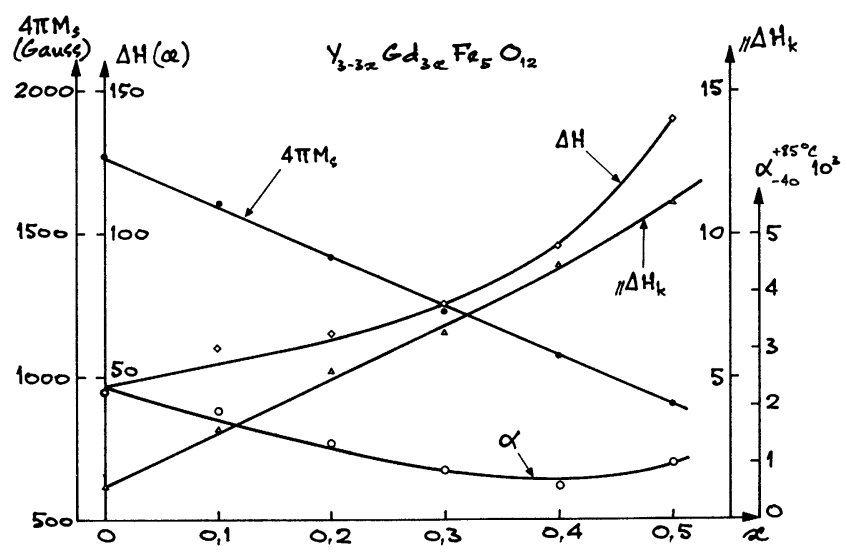

FIG. 7. - Propriétés des grenats classiques d'yttrium-gadolinium.
Le point de Curie reste sensiblement constant avec $x$ et le coefficient de température de $4 \pi M_{\mathrm{s}}$ diminue. Mais, comme le montre la figure $7, \Delta H$ et $\Delta H_{\mathrm{k}}$ s'accroissent.

Evidemment les deux moyens précédents peuvent être combinés pour réaliser des matériaux ayant des caractéristiques intermédiaires. On peut également augmenter l'aimantation jusqu'à une valeur de $4 \pi M_{\mathrm{s}}=1900 \mathrm{G}$ environ en substituant à des ions fer des ions indium non magnétiques, qui vont se loger dans les sites octaédriques. Cette augmentation de $4 \pi M_{\text {s }}$ est obtenue au détriment du point de Curie qui diminue et du coefficient de température de $4 \pi M_{\mathrm{s}}$ qui augmente. Par contre $\Delta H$ et $\Delta H_{k}$ restent faibles.

\subsection{TRAVAUX RÉCENTS EFFECTUÉS DANS LE DOMAINE} DES GRENATS POUR HYPERFRÉQUENCE. - On a cherché des matériaux ayant des caractéristiques améliorées par rapport à celles des matériaux classiques décrits au chapitre précédent. Deux voies ont été ouvertes dans des directions opposées qui nous semblent retenir l'attention.

5.2.1 Grenats polycristallins à très petit $\Delta H$. - Pour obtenir des ferrites polycristallins à très petit $\Delta H$ il faut partir de matériaux ayant une faible largeur de raie intrinsèque $\Delta H_{\text {int }}$ ce qui est facile car il suffit de n'avoir comme ions magnétiques que des ions fer trivalent. De plus il faut choisir un matériau ayant une très faible anisotropie magnétocristalline. Or on constate que dans les grenats des substitutions non magnétiques octaédriques telles que In ou $\mathrm{Zr}$ réduisent considérablement le champ équivalent d'anisotropie. Enfin il faut obtenir des matériaux très purs et homogènes exempts de porosité. Les progrès de la technologie ces dernières années ont été tels que l'on sait maintenant dans beaucoup de cas réduire la porosité des oxydes frittés à moins de un millième. Par ces moyens, des largeurs de raie $\Delta H$ aussi faibles que 1,5 Oe ont pu être obtenues sur des grenats polycristallins (Winkler). Le tableau II donne des caractéristiques mesurées sur de tels matériaux.

L'inconvénient de certains de ces matériaux à très faibles $\Delta H$ est d'avoir une assez mauvaise stabilité de leurs caractéristiques en fonction de la température. Des compromis techniques sont encore là à rechercher et les avantages que ces matériaux peuvent présenter dans la pratique à étudier maintenant en détail.

5.2.2 Grenats très stables en température. - On cherche à obtenir des courbes de $4 \pi M_{\mathrm{s}}$ en fonction de la température possédant un point de compensation.
Matériaux

(substitutions dans le grenat d'yttrium)

YIG pur

$\mathrm{Ca}, \mathrm{Zr}$

$\mathrm{Ca}, \mathrm{Ge}$, In

$\mathrm{Ca}, \mathrm{V}, \mathrm{In}$

TABLEAU II

$\begin{array}{ccc}4 \pi M_{\mathrm{s}} \mathrm{G} & \text { Point de Curie }{ }^{\circ} \mathrm{C} & \Delta H(\mathrm{Oe} \text { à } 9 \mathrm{GHz}) \\ \overline{1} 780 & \overline{2} & - \\ 1905 & 210 & 15 \\ 1500 & 130 & 4,2 \\ 1500 & 186 & 4,5 \\ & & 3,7\end{array}$

$\operatorname{tg} \delta_{\varepsilon}$ à $9 \mathrm{GHz}$

$$
\begin{gathered}
<10^{-4} \\
<10^{-4} \\
<10^{-4} \\
1,4 \times 10^{-4}
\end{gathered}
$$


La plupart des grenats des terres rares magnétiques possèdent une telle propriété comme l'a montré Pauthenet dès 1958. Cependant, les terres rares magnétiques sont des ions relaxants qui élargissent considérablement les largeurs de raie $\Delta H_{\text {int }}, \Delta H, \Delta H_{\text {eff }}$ et $\Delta H_{\mathrm{k}}$. Seul l'ion gadolinium $\mathrm{Gd}^{3+}$ ayant une couche $4 \mathrm{f}$ à moitié pleine (et donc pas de moment orbital) peut être introduit dans le matériau en proportion importante. Il provoque cependant un certain accroissement de $\Delta H_{\text {eff }}$ $(0,7$ Oe par \% de substitution d'ion $\mathbf{G d}$ dans les sites dodécaédriques) et de $\Delta H_{\mathrm{k}}(0,2$ Oe pour la même quantité). Ceci exclut dans les dispositifs l'obtention de très faibles pertes magnétiques mais favorise par contre une certaine tenue à la puissance hyperfréquence de crête. Pour ajuster $4 \pi M_{\mathrm{s}}$ et situer le maximum de la courbe de $4 \pi M_{\mathrm{s}}$ à la température voulue (ou minimiser le coefficient de température de $4 \pi M_{\mathrm{s}}$ ), on dispose des paramètres suivants :

a) le taux de substitution gadolinium,

b) des substitutions non magnétiques tétraédriques, telles que l'aluminium, qui produisent une diminution de $4 \pi M_{\mathrm{s}}$, une diminution du point de Curie et une augmentation du point de compensation,

c) des substitutions non magnétiques octaédriques, telles que l'indium ou l'étain, qui ont pour effet d'accroître $4 \pi M_{\mathrm{s}}$, de diminuer les points de Curie et de compensation. Ils réduisent aussi de manière très notable le champ équivalent d'anisotropie si bien qu'ils permettent l'obtention de largeurs de raie $\Delta H$ relativement faibles.

Les matériaux étudiés ont ainsi les compositions :

$$
\mathrm{Y}_{3-3 x-z} \mathrm{Gd}_{3 x} \mathrm{Ca}_{z} \mathrm{Fe}_{5-5 y-z} \mathrm{Al}_{5 y} \mathrm{Sn}_{z} \mathrm{O}_{12}
$$

$\mathrm{ou}$

$$
\mathrm{Y}_{3-3 x} \mathrm{Gd}_{3 x} \mathrm{Fe}_{5-5 y-z} \mathrm{Al}_{5 y} \mathrm{In}_{z} \mathrm{O}_{12} \text {. }
$$

Dans la première formule l'introduction de $\mathrm{Ca}^{2+}$ dans les sites dodécaédriques est destinée à compenser

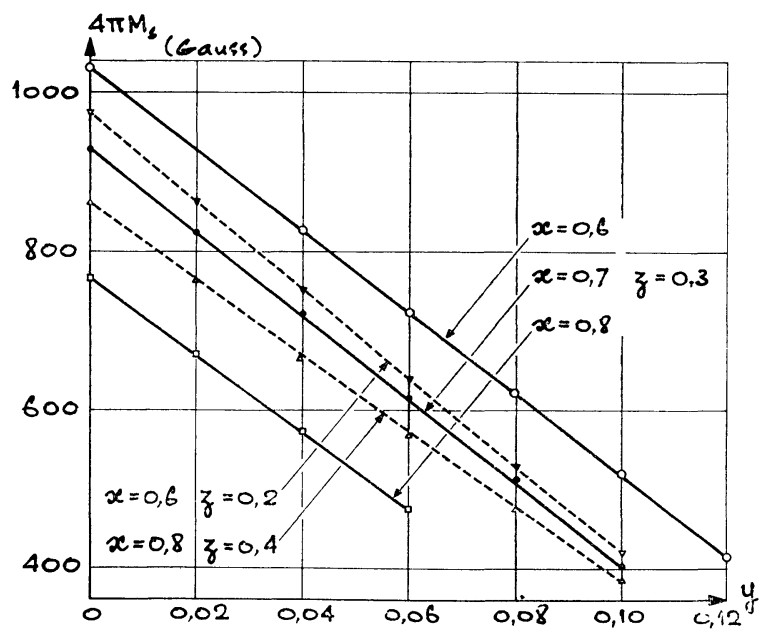

FIG. 8. - Aimantation $4 \pi M_{\mathrm{S}}$ en gauss à la température ambiante des grenats de composition

$$
\mathrm{Y}_{3-3 x-z} \mathrm{Gd}_{3 x} \mathrm{Ca}_{z} \mathrm{Fe}_{5-5 y-z} \mathrm{Al}_{5 y} \mathrm{Sn}_{z} \mathrm{O}_{12} \text {. }
$$

la valence excédentaire de $\mathrm{Sn}^{4+}$ tout en maintenant la totalité du fer à l'état trivalent. La figure 8 donne les aimantations à la température ambiante des grenats ayant la formule chimique (15). Des résultats pratiquement identiques sont trouvés avec ceux de la formule (16). La figure 9 montre à titre d'exemple

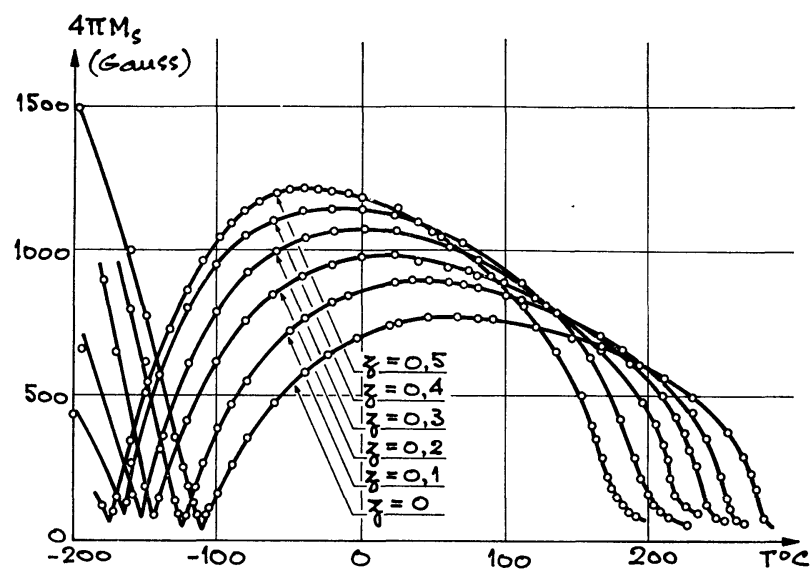

FIG. 9. - Influence sur les courbes d'aimantation $4 \pi M_{\mathrm{S}}$ en gauss en fonction de la température de substitutions non magnétiques octaédriques dans un grenat d'yttrium-gadolinium (de formule $\mathrm{Y}_{3-3 x-z} \mathrm{Gd}_{3 x} \mathrm{Ca}_{z} \mathrm{Fe}_{5-z} \mathrm{Sn}_{z} \mathrm{O}_{12}$ avec $\left.x=0,7\right)$.

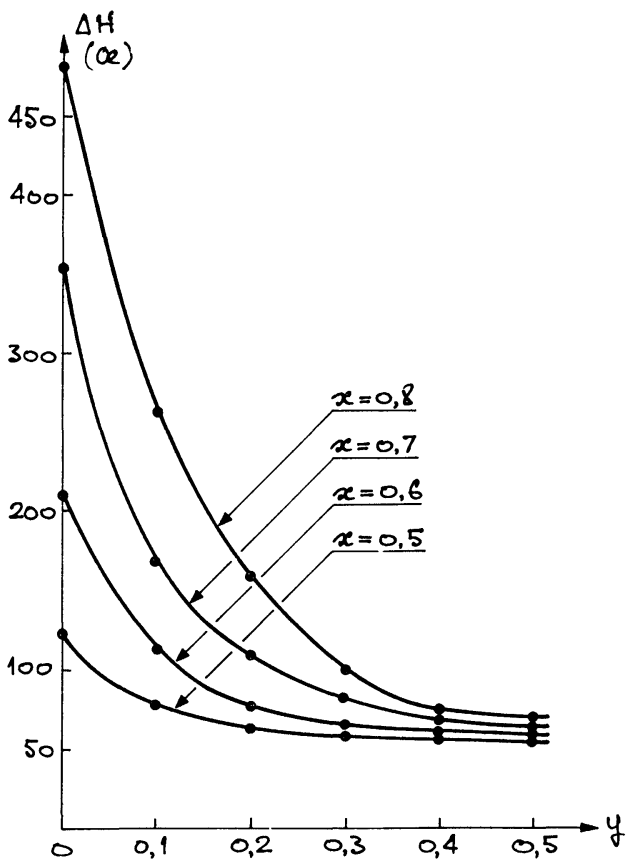

FIG. 10. - Influence de substitutions non magnétiques octaédriques sur la largeur de raie de résonance. Matériau de composition : $\mathrm{Y}_{3-3 x-z} \mathrm{Gd}_{3 x} \mathrm{Ca}_{z} \mathrm{Fe}_{5-z} \mathrm{Sn}_{z} \mathrm{O}_{12}$.

l'influence, sur la courbe d'aimantation en fonction de la température, de substitutions non magnétiques octaédriques. La figure 10 et la figure 11 montrent l'influence de substitutions de même nature sur les largeurs de raie $\Delta H$ et $\Delta H_{\mathrm{k}}$ à la température ambiante. 


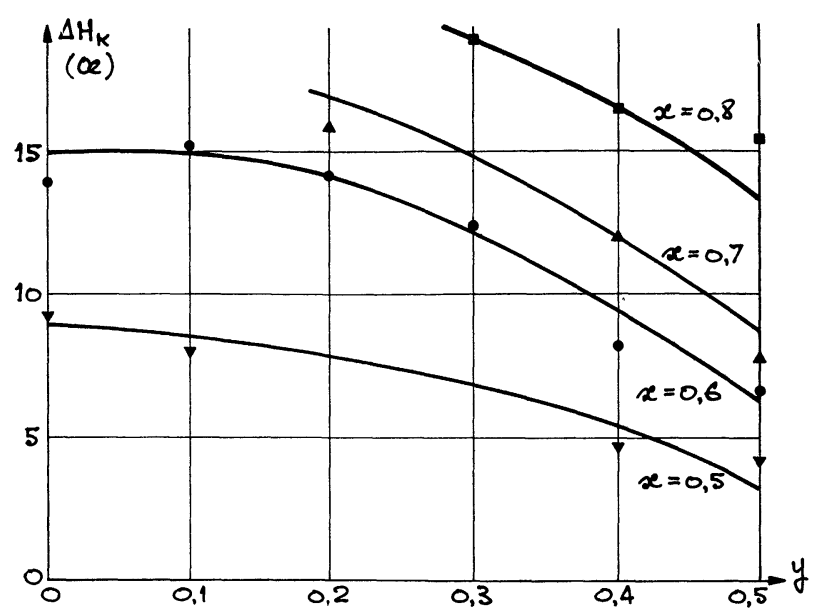

Fig. 11. - Largeur de raie d'ondes de spins $\Delta H_{\mathrm{k}}$ à la température ambiante des matériaux de composition :

$\mathrm{Y}_{3-3 x-z} \mathrm{Gd}_{3 x} \mathrm{Ca}_{z} \mathrm{Fe}_{5-2} \mathrm{Sn}_{z} \mathrm{O}_{12}$.

La figure 12 montre l'influence de substitutions non magnétiques tétraédriques sur la courbe de $4 \pi M_{\mathrm{s}}$ en température. La figure 13 donne la valeur des coefficients de température $\alpha_{-40}^{+85^{\circ}}$ de $4 \pi M_{\mathrm{s}}$ que l'on peut déduire de telles courbes. La figure 14 donne des valeurs de $\Delta H$ pour ce type de matériaux dont les $\Delta H_{\mathbf{k}}$ sont compris entre 10 et $20 \mathrm{Oe}$. Tous ces résultats sont donnés pour les matériaux de formule (15) mais ceux de formule (16) se comportent d'une manière très analogue.

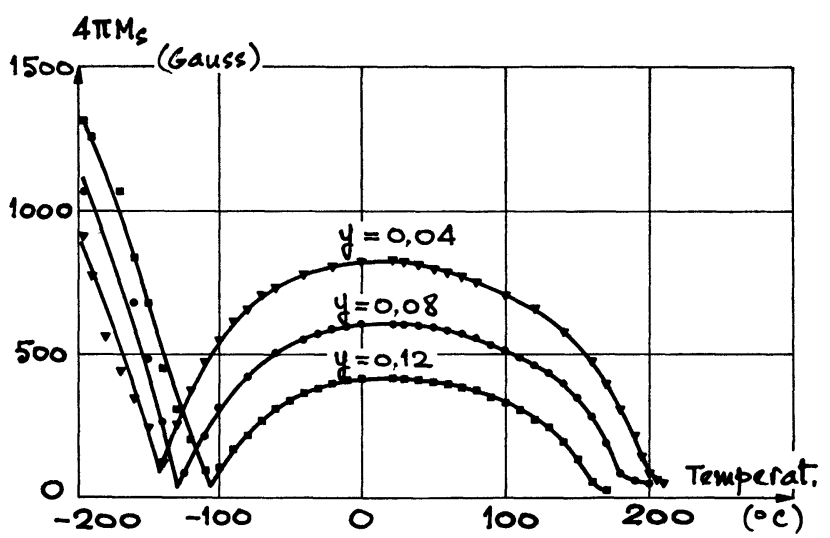

FIG. 12. - Influence sur les courbes d'aimantation en fonction de la température, de substitutions non magnétiques tétraédriques. Matériaux de composition :

$\mathrm{Y}_{3-3 x-z} \mathrm{Gd}_{3 x} \mathrm{Ca}_{z} \mathrm{Fe}_{5-5 y-z} \mathrm{Al}_{5 y} \mathrm{Sn}_{y} \mathrm{O}_{12}$ avec $x=0,6, \quad z=0,3 \cdot$

Ainsi on peut voir que pour des aimantations $4 \pi M_{\mathrm{s}}$ comprises entre 500 et $1000 \mathrm{G}$ des matériaux peuvent être créés, ayant des stabilités en température meilleures que les matériaux classiques tout en ayant des largeurs de raie $\Delta H$ assez faibles. Par contre ils ont des largeurs de raie d'ondes de spins $\Delta H_{\mathrm{k}}$ assez grandes, et ils ne conviennent pas aux applications à très faibles

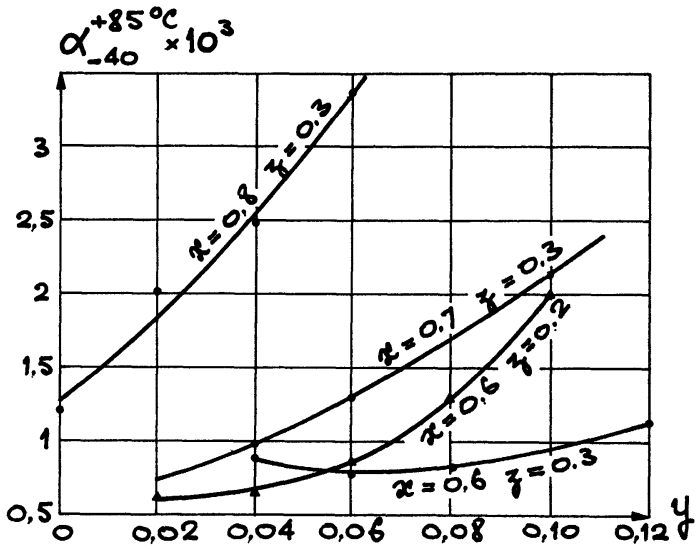

Fig. 13. - Coefficients moyens de température entre -40 et $+85^{\circ} \mathrm{C}$ des matériaux de composition :

$$
\mathrm{Y}_{3-3 x-2} \mathrm{Gd}_{3 x} \mathrm{Ca}_{z} \mathrm{Fe}_{5-5 y-z} \mathrm{Al}_{5 y} \mathrm{Sn}_{z} \mathrm{O}_{12} \text {. }
$$

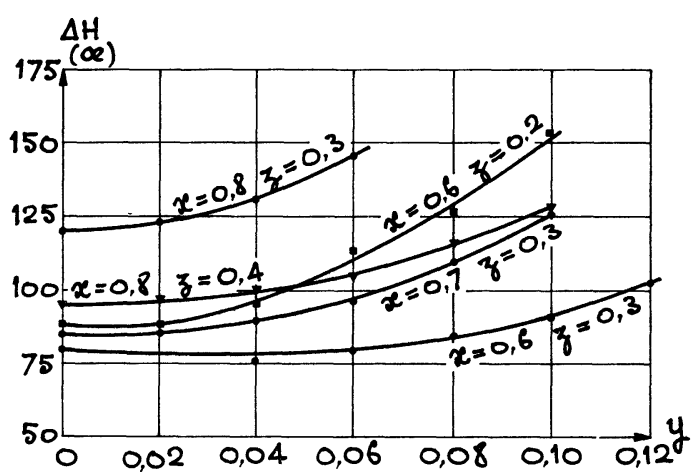

FIG. 14. - Largeurs de raie de résonance à la température ambiante de matériaux de composition :

$$
\mathrm{Y}_{3-3 x-z} \mathrm{Gd}_{3 x} \mathrm{Ca}_{z} \mathrm{Fe}_{5-5 y-z} \mathrm{Al}_{5 y} \mathrm{Sn}_{z} \mathrm{O}_{12} \text {. }
$$

pertes mais devraient être utiles dans celles où une tenue à de fortes puissances est nécessaire.

6. Ferrites de structure spinelle. - Les ferrites de structure spinelle prennent le relai des grenats pour les aimantations supérieures à 2000 G. Les diagrammes classiques les plus utilisés sont ceux des ferrites de manganèse-magnésium, des ferrites à base de nickel et ceux à base de lithium. Nous passerons en revue les propriétés de ces diagrammes.

6.1 FerRites DE MANGanèse-MAgnésium. - La composition chimique de base est :

$$
\mathrm{Mn}_{x} \mathrm{Mg}_{y} \mathrm{Fe}_{z} \mathrm{O}_{4} \text { avec } x+y+z \simeq 3 .
$$

Bien qu'historiquement apparus les premiers dans les applications hyperfréquences, ces ferrites sont assez complexes. L'ion $\mathrm{Mn}^{2+}$ a la même configuration électronique que $\mathrm{Fe}^{3+}$. Mais une partie du manganèse peut exister à l'état de $\mathrm{Mn}^{3+}$. D'autre part les ions métalliques se répartissent entre les deux sites tétraédriques et octaédriques d'une manière qui dépend du traitement thermique appliqué ; ainsi $x, y$ et $z$ sont déterminés expérimentalement, en fonction du traite- 
ment de synthèse pour optimiser les caractéristiques. On obtient des aimantations de 2000 à $2500 \mathrm{G}$ avec des points de Curie entre 270 et $300^{\circ} \mathrm{C}$. Les coefficients de températures de $4 \pi M_{\mathrm{s}}$ sont relativement importants : $\left(\alpha_{-40}^{+85} \simeq 2,6 \times 10^{-3}\right)$. Les largeurs de raies $\Delta H$, dues surtout à l'élargissement par l'anisotropie sont de 300 à 400 Oe tandis que les largeurs de raie d'ondes de spins sont faibles, de l'ordre de 3,5 Oe. Ces matériaux conviennent donc bien à des applications à bas niveau de puissance hyperfréquence, sans conditions sévères de température à des fréquences de 7000 à $10000 \mathrm{MHz}$. Il est possible d'abaisser l'aimantation en substituant une partie du fer par de l'aluminium au détriment du point de Curie et du coefficient de température.

6.2 FERrites DE NICKEL. - Le ferrite de nickel $\mathrm{NiFe}_{2} \mathrm{O}_{4}$ a un point de Curie de l'ordre de $570{ }^{\circ} \mathrm{C}$ et son aimantation $\left(4 \pi M_{\mathrm{s}}=3200 \mathrm{G}\right)$, est donc beaucoup plus stable en température que celle des matériaux précédents $\left(\alpha_{-40}^{+85} \simeq 0,43 \times 10^{-3}\right)$, mais l'ion nickel possédant un moment magnétique orbital est un ion relaxant, si bien que le ferrite de nickel ne convient pas pour les dispositifs à très faibles pertes d'insertion mais plutôt à ceux devant tenir une certaine puissance de crête. L'effet de l'anisotropie magnétocristalline sur $\Delta H$ du ferrite de nickel est assez important mais il peut être réduit par de faibles substitutions de $\mathrm{Co}^{2+}$ dont l'anisotropie est très grande et de signe contraire à celle des ions fer. Cet ion cobalt accroît encore $\Delta H_{\mathbf{k}}$. On arrive ainsi à une composition du type $\mathrm{N}_{1-x} \mathrm{Co}_{x} \mathrm{Fe}_{2} \mathrm{O}_{4}$ (avec $x \simeq 0,02$ ). Il est toutefois, pour des raisons physicochimiques, difficile d'obtenir un produit dense et sans pertes diélectriques. Pour diminuer autant que possible ces dernières, on pratique des additions de quelques pour cent de manganèse. Finalement on arrive à un matériau avec $\Delta H=250 \mathrm{Oe}, \Delta H_{\mathrm{k}}=26 \mathrm{Oe}$ et $\operatorname{tg} \delta_{\varepsilon}<10^{-3}$, toutes ces grandeurs étant mesurées à $10000 \mathrm{MHz}$. Des substitutions d'aluminium au fer réduisent là encore l'aimantation, tandis que des substitutions de zinc l'augmentent jusque vers $4800 \mathrm{G}$. Dans les deux cas la température de Curie est abaissée.

6.3 FerRites DE LiTHIUM. - Le ferrite de lithium $\mathrm{LiFe}_{5} \mathrm{O}_{8}$ a une aimantation de l'ordre de $4 \pi M_{\mathrm{s}}=3700 \mathrm{G}$ avec un point de Curie élevé, voisin de $645^{\circ} \mathrm{C}$, si bien qu'il a une stabilité en température analogue à celle du ferrite de nickel. Il est intéressant de remarquer que comme le grenat d'yttrium, c'est un corps qui ne possède comme ions magnétiques, que des ions fer trivalent. Il en résulte une très faible largeur de raie d'ondes de spins $\Delta H_{\mathrm{k}}$ de l'ordre de 2,5 Oe et donc aussi une faible largeur de raie effective $\Delta H_{\text {eff }}$. Cela prédispose les ferrites à base d'ions lithium (substitués éventuellement pour ajuster l'aimantation entre 1000 et $5000 \mathrm{G}$ ) aux utilisations à faible niveau de puissance hyperfréquence et faible perte d'insertion. Mais lorsque l'on veut réaliser de tels matériaux on est confronté à deux problèmes :

a) L'anisotropie magnétocristalline du ferrite de lithium est relativement importante et conduit à une largeur de raie $\Delta H$ de l'ordre de 400 Oe.

b) Les matériaux sont industriellement difficiles à faire de manière reproductible avec une bonne densité, de bonnes pertes diélectriques. Ceci est dû en partie à la volatilité de l'oxyde de lithium.

Des solutions ont été trouvées dans notre laboratoire et aussi aux Etats-Unis par Baba, pour tourner ces difficultés. Il y a lieu d'abord de choisir les compositions pour abaisser autant que possible la température de frittage de ces composés de manière à minimiser sinon annuler le départ d'oxyde de lithium dans la phase vapeur. Pour cela, outre l'utilisation de certains fondants on choisit comme ions non magnétiques de substitution octaédrique les ions $\mathrm{Ti}^{4+}$ plutôt que les ions $\mathrm{Al}^{3+}$ qui ont tendance à nécessiter une température de synthèse plus élevée. La valence excédentaire de $\mathrm{Ti}^{4+}$ est compensée par un excès de $\mathrm{Li}^{+}$pour maintenir la totalité du fer à l'état trivalent. Certaines additions comme celles d'oxyde de manganèse améliorent les pertes diélectriques qui peuvent être ramenées d'une manière reproductible au niveau de $\operatorname{tg} \delta_{\varepsilon}=2$ à $4 \times 10^{-4}$. Des substitutions de zinc dans les sites tétraédriques augmentent l'aimantation et diminuent le champ équivalent d'anisotropie. Elles permettent donc la réduction de $\Delta H$. Evidemment les substitutions de $\mathrm{Ti}$, Li ou de $\mathrm{Zn}$ abaissent le point de Curie. Mais on peut dire dès maintenant que des compromis techniques peuvent être trouvés mettant en jeu la valeur de l'aimantation, sa variation en température et la valeur de $\Delta H$ qui dépassent ceux que l'on peut obtenir dans les diagrammes des ferrites de manganèse-magnésium.

Les faisceaux hertziens, qui constituent le plus grand débouché pour les ferrites hyperfréquences, utilisent actuellement surtout des grenats. La tendance à augmenter les fréquences au fur et à mesure que le réseau de télécommunication se densifie doit logiquement conduire à remplacer les grenats par des ferrites à base de lithium. On devrait donc dans les années qui viennent voir se créer une gamme complète de ces matériaux aux applications de plus en plus nombreuses.

7. Conclusion. - Les ferrites sont devenus irremplaçables dans la technique des hyperfréquences, particulièrement dans les radars et les faisceaux hertziens. Ces applications ont exigé des matériaux de plus en plus performants et en fait des améliorations ont été faites continuellement au cours du temps. Les pertes diélectriques ont été réduites dans la plupart des cas à une quantité presque négligeable. L'effet sur les pertes magnétiques de l'amortissement magnétique a été beaucoup mieux compris, particulièrement par l'introduction de la largeur de raie effective $\Delta H_{\text {eff }}$. Cette dernière a été trouvée logiquement reliée à la largeur de raie d'ondes de spin $\Delta H_{\mathrm{k}}$. Ces deux grandeurs doivent traduire en effet de manière différente le même phénomène élémentaire d'amortissement des mouvements de spins. Compte tenu de la connaissance détaillée que 
l'on a des effets non linéaires, des dispositifs à haute tenue à la puissance crête ont pu être réalisés en utilisant des matériaux judicieusement choisis à faibles pertes magnétiques en jouant sur le champ statique de polarisation. Ces progrès ont pu être réalisés en grande partie grâce aux améliorations intervenues dans la caractérisation fine des matériaux en cause.

Des grenats polycristallins ayant des largeurs de quelques oersteds peuvent être maintenant obtenus. D'autre part avec des substitutions complexes dans les trois sous-réseaux magnétiques des grenats on peut créer des matériaux dont l'aimantation est très stable en fonction de la température et qui possèdent néanmoins des largeurs de raie relativement faibles. Une nouvelle classe de spinelles de lithium apparait qui prendra vraisemblablement le relai des grenats pour les utilisations aux plus hautes fréquences ( $>5000 \mathrm{MHz})$.

Certaines questions ayant un aspect plus scientifique que technique posent toujours des problèmes. On peut citer l'explication détaillée des phénomènes d'amortis- sement magnétique, soit à l'échelle microscopique, soit à une échelle "intermédiaire ", comme se situe par exemple l'influence de la taille des cristallites sur $\Delta H_{\text {eff }}$ et $\Delta H_{\mathrm{k}}$. On peut citer également la maîtrise pratique de l'anisotropie magnétocristalline qui est loin d'être satisfaisante et pour laquelle des progrès seraient les bienvenus. Enfin, on peut évoquer le problème des matériaux non magnétiquement saturés. Ils possèdent une susceptibilité magnétique moyenne dépendant de l'état d'aimantation. Une des difficultés majeures pour bien comprendre cette susceptibilité moyenne est qu'elle nécessite la connaissance du champ agissant en chaque point du matériau. C'est spécialement le champ d'origine dipolaire en présence des parois de domaines qu'il est difficile d'appréhender.

Ainsi, le domaine des ferrites pour hyperfréquences apparaît comme très riche et largement ouvert. Il est probable qu'il sera encore l'objet de perfectionnements et de développements notables dans les proches années à venir.

\section{Bibliographie}

\section{OUVRAGES GÉNÉRAUX}

[1] Sооноо, R. F., Theory and application of ferrites (Prentice Hall, USA) 1960.

[2] LAX, B. and ButToN, K. J., Microwave ferrites and ferrimagnetics (McGraw Hill Book Company, New York) 1962.

[3] Von Aulock, W. H., Handbook of microwave ferrite materials (Academic Press New York) 1965.

[4] SPAKs, M., Ferromagnetic Relaxation Theory (McGraw Hill Book Company, New York) 1964.

[5] PIRCher, G., Ferrites et grenats. Phénomènes non linéaires (Dunod) 1969.

[6] Von Aulock, W. H. and FAY, C. E., Linear ferrite devices for microwave applications (Academic Press, New York) 1968.

\section{ARTICLES GÉNÉRAUX SUR FERRITES HYPERFRÉQUENCES}

[7] Nicolas, J., Lagrange, A., Journée DRME 1969.

[8] Deschamps, A., Câbles et Transmissions no 4 (1970) pp. 357 359.

[9] Schlömann, E., J. Physique Collq. 32 (1971) C1 443-451.

[10] Nicolas, J., Lagrange, A., Sroussi, R., Inglebert, R. L., IEEE Transactions on Magnetics MAG-9 (1973) 546-551.

\section{PROPRIÉTÉS DIÉLECTRIQUES}

[11] Hermosin, A., Thèse $3^{\text {e }}$ cycle. Orsay 1970.

\section{FERRIMAGNÉTISME}

[12] Néel, L., Annls de Phys. 3 (1948) 137-268.

[13] Pauthenet, R., Thèse Grenoble 17 mars 1951.

[14] Pauthenet, R., Thèse Grenoble 1958.

RÉSONANCE MAGNÉTIQUE, $\Delta H, \Delta H_{\text {eff }}$

[15] Wangsness, R. K., Phys. Rev. 91 (1953) 1085-1091.

[16] Bloembergen, N., Proceedings of the IRE (1956) 1259-1269.
[17] Schlömann, E., A IEEE Spec. Pub. Proc. Conf. Magnetism and Magnetic Materials 91 (1956) 600.

[18] Patton, C. E., Phys. Rev. 179 (1969) 352-358.

[19] Vrehen, Q. H. F., J. Appl. Phys. 40 (1969), 1849-1860.

EFFETS NON LINÉAIRES. ONDES DE SPINS. RELAXATION MAGNÉTIQUE

[20] Harry SuHL, Proceedings of IRE (1956) pp. 1270-1284.

[21] Kittel, C., Phys. Rev. 115 (1959).

[22] De Gennes, P. G., Kittel, C., Portis, A. M., Phys. Rev. 116 (1959).

[23] Schlöman, E., Green, J. J., Milano, U., J. Appl. Phys. 31 (1960) 386S-395S.

[24] Schlömann, E., Joseph, R. I., J. Appl. Phys. 32 (1961).

[25] Patton, C. E., IEEE Trans. Magn. MAG-8 (1972) 433-39.

[26] Hartmann-Boutron, F., Phys. Kondens. Materie 2 (1964) 80-98.

[27] Hartmann-Boutron, F., C. R. Hebd. Séan. Acad. Sci. 259 (1964) 2085.

[28] Borghèse, C. et Roveda, R. L., J. Physique Collq. 32 (1971) C1 150-152.

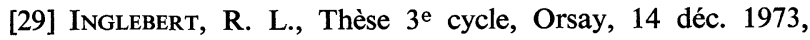

\section{GRENATS}

[30] Bertaut, F. et Forrat, F., C. R. Hebd. Séan. Acad. Sci. 242 (1956) $382-84$

[31] Geller, S., J. Appl. Phys. 31 (1960) 30S-37S.

[32] Geller, S., Williams, H. J., Espinosa, G. P. and Sherwood, R. C., Phys. Rev. 136 (1964) A 1650-A 1656.

[33] Vassiliev, A., Nicolas, J., Hildebrandt, M., C. R. Hebd. Séan. Acad. Sci. 252 (1961) 2529-2531.

[34] Vassiliev, A., Nicolas, J., Z. Angew. Phys. 18 (1965) S 557560 .

[35] Nicolas, J., Lagrange. A., Sroussi, R., IEEE Transactions on Magnetics MAG-6 (1970) 608-610.

[36] Nicolas, J., LAGRANGe, A., Proceedings of the International Conference July 1970, Japan, pp. 527-529. 
[37] Winkler, G., Hansen, P., Holst, P., Philips Research Repts. 27 (1972) 151-171.

[38] Sroussi, R., Nicolas, J., Intermag 1974 (à paraître).

\section{FERRITES DE NICKEL}

[39] Gorter, E. W., Philips Res. Rev. 9 (1954).

[40] Maxwell, L. R., Pickart, S. J., Phys. Rev. 92 (1953) 1120.

[41] Nicolas, J., Thèse Paris février 1966.

[42] Nicolas, J., J. Phys. \& Chem. Solids 28 (1967) 847-859.

\section{FERRITES DE LITHIUM}

[43] VASSiliev, A., Thèse Paris octobre 1962.

[44] Vassiliev, A., Lagrange, A., IEEE Trans. on Magn. MAG-2 (1966) 707-710.

[45] Baba, P. D., Argentina, G. M., Courtey, W. E., Dionne, G. F., Temme, D. H., IEEE Trans. on Magn. MAG-8 (1972) 83-94.
[46] Dionne, G. F., Temme, D. H., IEEE Trans. on Magn. MAG-8 (1972) 83-94.

FERRITES HYPERFRÉQUENCES PRESSÉS A CHA UUD

[47] Nicolas, J., Lagrange, A., Sroussi, R., Hildebrandt, M., Revue Technique Thomson-CSF 1 (1969) 505-522.

[48] Igarashi, H., OKAZAKI, K., Proceedings of the International Conference, July 1970, Japan, pp. 530-532.

[49] Nicolas, J., HildeBrandT, M., " Proceedings of the sixth international Conf. on Science of ceramics» Published by (Deutsche Keramiche Gesellschaft) 1973 XXX pp. 1-16.

COMPORTEMENT AUX HYPERFRÉQUENCES DES FERRITES NON MAGNÉTIQUEMENT SATURÉS

[50] Nicolas, J., Annales de Radioélectricité XXII (1967) 109120.

[51] Courtois, L., Deschamps, A., C. R. Hebd. Séan. Acad. Sci. 264 (1967) 1333.

[52] Schlömann, E., J. Appl. Phys. 41 (1970) 204-214. 